Title: DEVELOPMENT OF A FAST TRAVELING-WAVE BEAM CHOPPER FOR THE NATIONAL SPALLATION NEUTRON SOURCE

$\begin{array}{cl}\text { Author(s): } & \text { Sergey S. Kurennoy } \\ \text { Andrew J. Jason } & \text { LANSCE-1 } \\ \text { Frank. L. Krawczyk } & \text { LANSCE-1 } \\ \text { John F. Power } & \text { LANSCE-1 } \\ & \text { LANSCE-1 }\end{array}$

RECEIVED

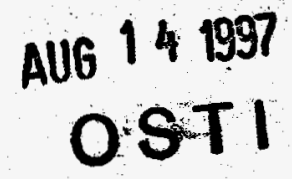

Submitted To: 1997 Particle Accelerator Conference

Vancouver, BC, Canada

May 12-16, 1997

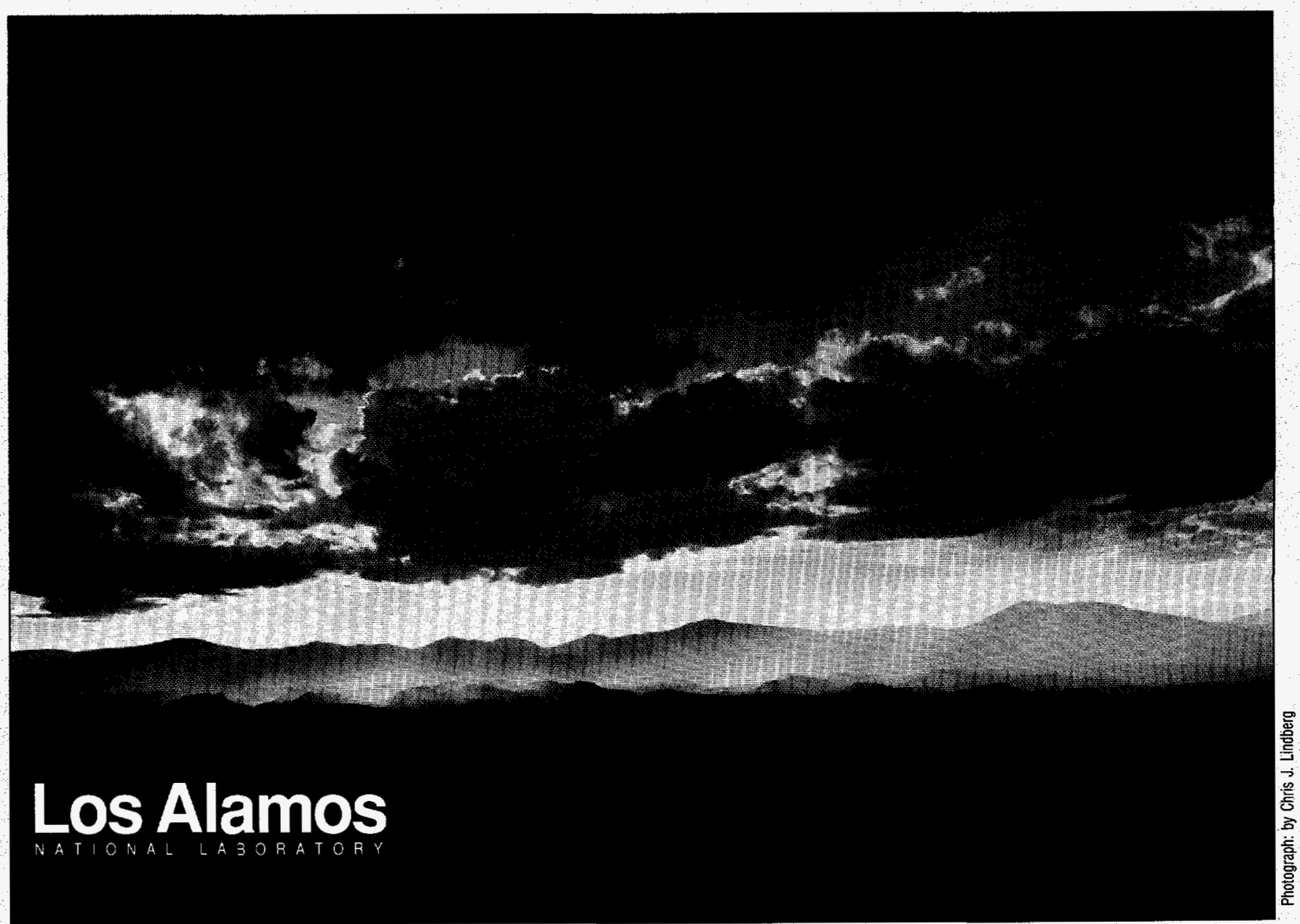

This is a preprint of a paper intended for publication in a joumal or proceedings. Because changes may be made belore publication, this preprint is made available with the understainding that it will not be cited or reproduced without the permission of the author. 


\title{
DEVELOPMENT OF A FAST TRAVELING-WAVE BEAM CHOPPER FOR THE NATIONAL SPALLATION NEUTRON SOURCE ${ }^{*}$
}

\author{
Sergey S. Kurennoy, Andrew J. Jason, Frank L. Krawczyk, and John Power, \\ LANSCE-1, Los Alamos National Laboratory, Los Alamos, NM 87545, USA
}

\begin{abstract}
High current and severe restrictions on beam losses, below $1 \mathrm{nA} / \mathrm{m}$, in the designed linac for the National Spallation Neutron Source (NSNS) require clean and fast - with the rise time from $2 \%$ to $98 \%$ less than $2.5 \mathrm{~ns}$ to accommodate a $402.5-\mathrm{MHz}$ beam structure - beam chopping in its front end, at the beam energy $2.5 \mathrm{MeV}$. The $R \& D$ program includes both modification of the existing LANSCE coaxplate chopper to reduce parasitic coupling between adjacent plates, and development of new traveling-wave deflecting structures, in particular, based on a meander line. Using analytical methods and three-dimensional time-domain computer simulations we study transient effects in such structures to choose an optimal chopper design.
\end{abstract}

\section{REQUIREMENTS}

The NSNS, to be constructed at Oak Ridge National Laboratory, is a next-generation pulsed spallation neutron source designed to deliver $1 \mathrm{MW}$ of beam power on the target at $60 \mathrm{~Hz}$ in its initial stage [1]. It consists of a $1-\mathrm{GeV}$ 28-mA linear $\mathrm{H}^{-}$accelerator and an accumulator ring. The NSNS project is a collaboration of 5 national laboratories. Los Alamos National Laboratory is responsible for the linac design and the chopping system of its front end.

Beam injected into the NSNS accumulator ring must be chopped at near the ring-rotation frequency $1.189 \mathrm{MHz}$ in order to leave a gap for the kicker rise time during oneturn ring extraction. The duty factor of the injected beam has been set at 0.65 . Chopping is to be done in the Medium Energy Beam Transport (MEBT) line that transports $28-\mathrm{mA}$ of peak beam from a $2.5-\mathrm{MeV} 402.5-\mathrm{MHz}$ RFQ to a drifttube linac. A $0.5-\mathrm{m}$ space is currently allocated for the chopper that deflects beam into a collimator during the $35 \%$ beam-off time. In case of a partial chopping or small errors in the timing system, an identical "anti-chopper" is placed in the line at an optically symmetric point from the chopper in order to return uncollimated beam to the axis.

Using a planar structure, with a half gap of $1 \mathrm{~cm}$ and a $900-\mathrm{V}$ deflection voltage on each plate, the chopper will provide a beam deflection of $9 \mathrm{mrad}$. The gap between plates can be further reduced, down to a half gap of $7.5 \mathrm{~mm}$ or even $5 \mathrm{~mm}$. A small tilt of the chopper structure in the vertical plane will be required in the last case to avoid beam hitting the plates. A traveling-wave structure, with the pulse

\footnotetext{
- This work is supported by the US Department of Energy under contract DE-AC05-960R22464.
}

\section{MASTEK}

phase velocity along the beam path matching the beam velocity $(\beta=0.073)$, will be used to avoid transit-time effects. The chopper is required to rise from $2 \%$ to $98 \%$ (and fall) in $2.5 \mathrm{~ns}$ to accommodate the $402.5-\mathrm{MHz}$ beam structure. Partial chopping of a micropulse would cause decreased charge in the transmitted pulse with subsequent variation of the linac-output Twiss parameters and increased loss in the transport line and ring.

An additional function of the chopper is pulse width modulation of the beam. At the start of the macropulse, it is necessary to increase the beam intensity from zero to full to accommodate linac beam loading. This is done by decreasing the width of the chopper deflection pulse from full to nominal $35 \%$. This function can be also used in experiments that require fractional ring filling or simply to modulate beam intensity.

\section{PRESENT STATUS}

The present chopper system [2] at Los Alamos Neutron Science Center (LANSCE) was constructed for the Proton Storage Ring (PSR). It works at the beam energy $750 \mathrm{keV}$ and provides the rise time of approximately $7 \mathrm{~ns}$ with a larger contribution from the pulse modulator. The current structure itself is capable of providing a pulse front slightly longer than $2 \mathrm{~ns}$, with an overshoot on the $10 \%$ level ringing for a few nanoseconds. This coax-plate structure is $1-\mathrm{m}$ long and consists of two parallel plates each interfaced with many small strips which are connected with coaxial cables on the reverse side of each plate to form a circuit that is continuous along the structure (Fig. 1).

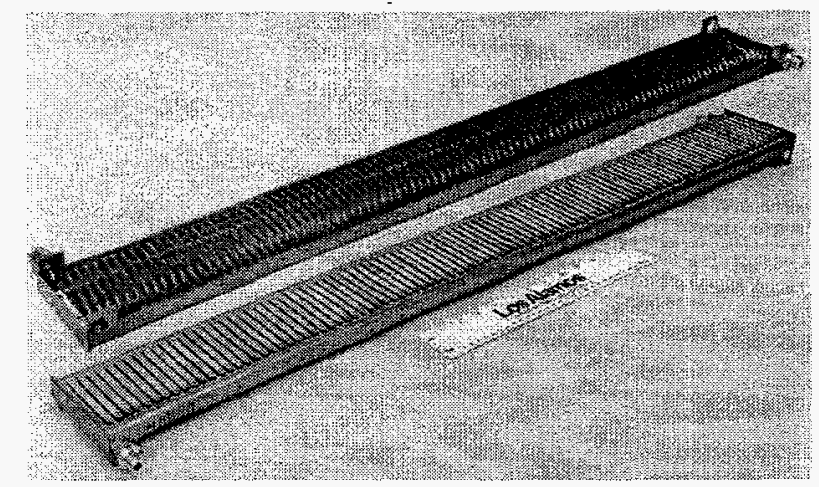

Figure 1: Photograph of the traveling-wave chopper structure used at LANSCE. 


\section{DISCLAMIER}

Portions of this document may be illegible in electronic image products. Images are produced from the best available original document. 


\section{DISCLAIMER}

This report was prepared as an account of work sponsored by an agency of the United States Government. Neither the United States Government nor any agency thereof, nor any of their employees, make any warranty, express or implied, or assumes any legal liability or responsibility for the accuracy, completeness, or usefulness of any information, apparatus, product, or process disclosed, or represents that its use would not infringe privately owned rights. Reference herein to any specific commercial product, process, or service by trade name, trademark, manufacturer, or otherwise does not necessarily constitute or imply its endorsement, recommendation, or favoring by the United States Government or any agency thereof. The views and opinions of authors expressed herein do not necessarily state or reflect those of the United States Government or any agency thereof. 
The voltages on the upper and lower plates are synchronized and have opposite signs so that the resulting vertical electric field deflects the beam.

\section{STRUCTURE DEVELOPMENT}

\subsection{Coax-plate structure}

The major limitations on the structure rise time for the existing coax-plate design are caused by stray capacitance between the electrode segments and by multiple coax-toplate transitions. A similar structure for the higher energy, $2.5 \mathrm{MeV}$, would have slightly better rise and fall times, simply because one can use wider strips and make wider gaps between strips in the structure to match the higher beam velocity. Wider gaps reduce strip-to-strip coupling. As an additional way to reduce this parasitic coupling between adjacent strips, guard barriers (or separators) rising from the ground plane have been proposed.

\subsection{Meander-line structure}

A promising alternative design under consideration is a meander line with separators, see Fig. 2, instead of the coax-plate current structure. Such a design has no transitions, and the meander bends can be properly chamfered to avoid pulse reflections. It is also easier to manufacture. The meander line parameters are calculated analytically to match the beam velocity and provide the line characteristic impedance $Z_{c}=50 \Omega$. It results in the strip width $w=5 \mathrm{~mm}$, strip-to-strip gap $g=2 \mathrm{~mm}$, the full meander width $b=(w+g)(1 / \beta-1)=89 \mathrm{~mm}$, and the distance between strips and the ground plate $h=1 \mathrm{~mm}$. The chamfering cuts are triangles $w \times w \times w \sqrt{2}$. For structures with separators, $h$ should be slightly increased, to $1.25 \mathrm{~mm}$ for the separators as high as $h$, to keep $Z_{c}$ fixed.

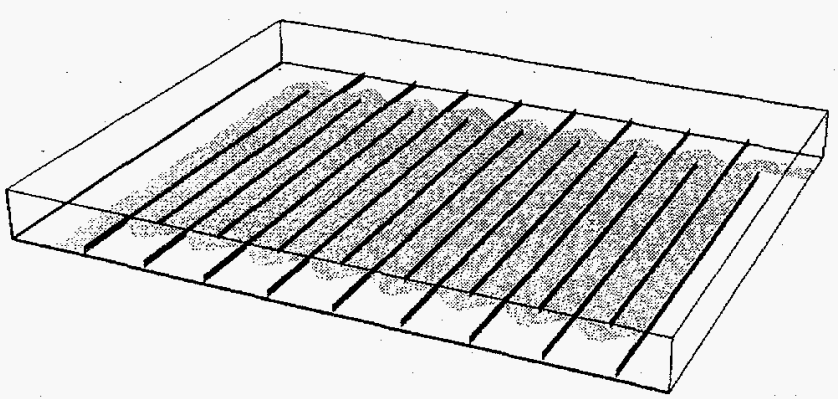

Figure 2: The meander structure (one quarter of the total length) with separators (dark) used in MAFIA simulations.

Transient effects in meander structures have been studied thoroughly using time-domain 3D simulations with the code package MAFIA [3]. The structure is fed with a TEM-wave pulse whose amplitude can be switched on as an arbitrary function of time, e.g., an abrupt or smoothed step, or a pulse of a finite time-length. It allows us to simulate any modulator pulse voltage. The pulse propagates along the strip and its vertical electric field on the beam path is recorded as a function of time. Figure 3 presents results of transient-effects simulations in the structure with the length of about $25 \mathrm{~cm}$ along the beam, one half of the full chopper length. The signal amplitude versus time in this case was a slightly smoothed step (squared sin with the full rise in 0.15 ns). It can be seen that even without separators the meander structure can provide a rise time about 2-2.5 ns, while with separators the rise time can be reduced down to $1-1.25 \mathrm{~ns}$, depending on the separator height.

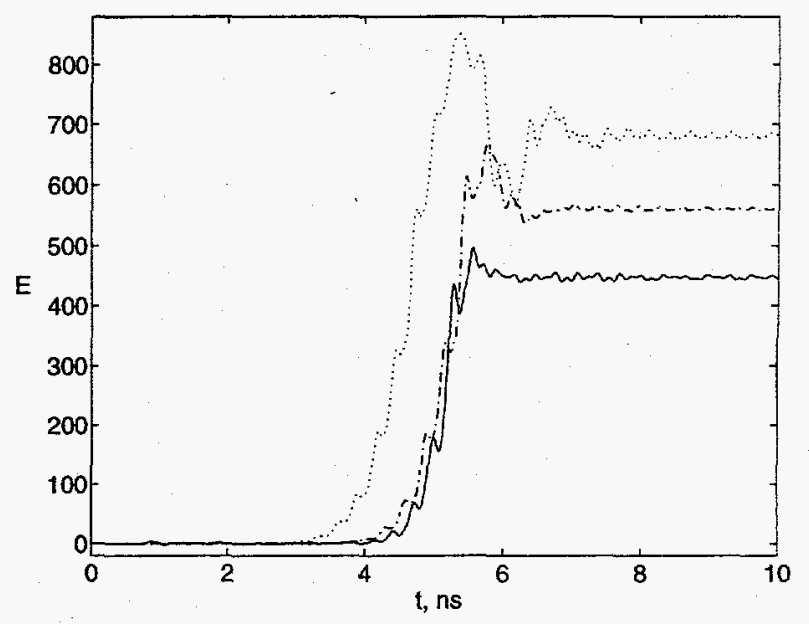

Figure 3: Vertical electric field, in arbitrary units, at a fixed point on the beam path versus time in the half-length meander structure with separators $h_{s}=2 h$ (solid), $h_{s}=h$ (dash-dotted), and without them (dotted).

Simulations have also been performed for meander structures having small low-dielectric $(\varepsilon=2-4)$ posts used as supports. The support influence was found negligible.

The structure efficiency $\eta$ can be defined as a ratio of the deflecting field to that produced by continuous plates under the same voltage. The designed meander line without separators has $\eta=0.94$. The separators reduce the effective field on the beam path, as one can see in Fig. 3. The $\eta$ decrease can also be easily calculated by solving an electrostatics problem; it depends on the separator height $h_{s}$, being about $10-12 \%$ for $h_{s}=h$ and almost $20-25 \%$ for $h_{s}=$ $2 h$. This should be compensated either by reducing the gap or by increasing the voltage.

We have completed the transient-effect simulations for the quarter-length, half-length, and the full-length current structure $(50 \mathrm{~cm}$ long), including simulations with a finite pulse rise time. The results are similar to those shown in Fig. 3, except that the pulse slightly deteriorate closer to the end of the long structure, mostly by developing some ringing overshoot about 1-2 ns long. The structure fall time was found in all simulations to be about the same as the rise time. Figure 4 shows the propagation of a 4-ns long pulse along the quarter-length meander structure. One can see a small overshoot developing as the pulse reaches the 
structure end. The front and back pulse edges remain well within $1 \mathrm{~ns}$.

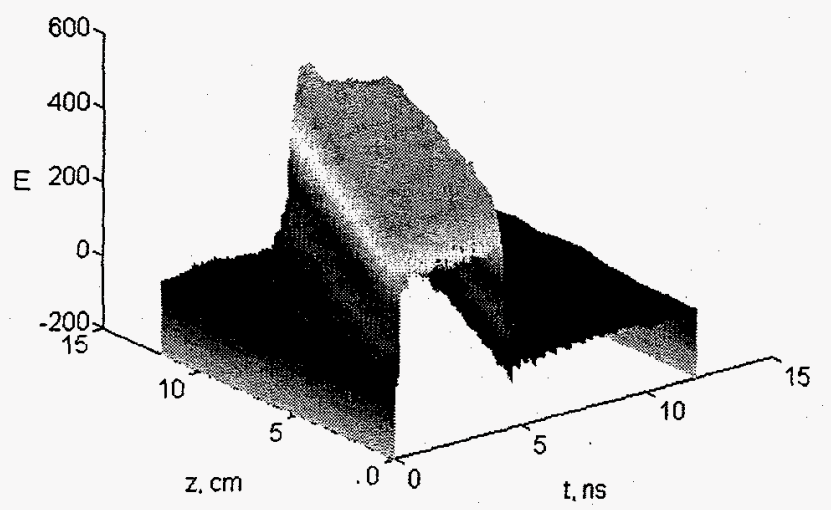

Figure 4: Vertical electric field at the beam path of a 4-ns long pulse propagating along in the meander structure.

To check our simulation results, a simple model structure with a meander line on a printed-circuit board (PCB) has been manufactured at LANL. The PCB with the meander on its bottom surface was placed on $1.25-\mathrm{mm}$ barriers-separators sticking out of the aluminum ground plane. The prototype length is about $12 \mathrm{~cm}$. The transient effects in the prototype have been measured with a TDR and were found to agree well with the MAFIA simulations for that case, with all transitions within a 2-ns range.

\subsection{Notched meander-line structure}

Another way to increase the efficiency is to use a notched strip line in the meander instead of a straight one [4]. The magnetic field of the wave propagating along the notched strip is concentrated closer to the strip center, and the wave phase velocity is less then $c$ due to the inductive loading by notches.

The design reduces a strip-to-strip magnetic coupling. It also allows us to have a larger ratio of the strip width to the width of the gap between adjacent strips, which increases the efficiency $\eta$. On the other hand, the notched line has some additional dispersion that can worsen the rise and fall times. Analytical calculations and simulations of the notched-strip meander structure are underway.

\section{PULSE GENERATOR}

The LANSCE pulse modulator was originally a vacuum-tube-driven device. It has been revised to a solidstate model, easier to maintain at peak performance, but with a slower rise time. The modulator rise time is largely limited by semiconductor limitations and inaccuracies in matching. It appears likely that the fastest speeds possible with the present-day FET technology will be on the order of $4 \mathrm{~ns}$. This speed limitation is largely independent of voltage and limited to around $900 \mathrm{~V}$ across $50 \Omega$.
The main problem is to satisfy the requirements of the fast pulse fronts, high repetition rate, and high voltage simultaneously. A modest program with semiconductor manufacturers for development of fast FET semiconductors is believed necessary. A simplified schematic of the proposed pulser design is shown in Fig. 5. It uses a combination of two switches, one to generate a fast rise time and a second to clamp the end of the output pulse to provide a faster fall time.
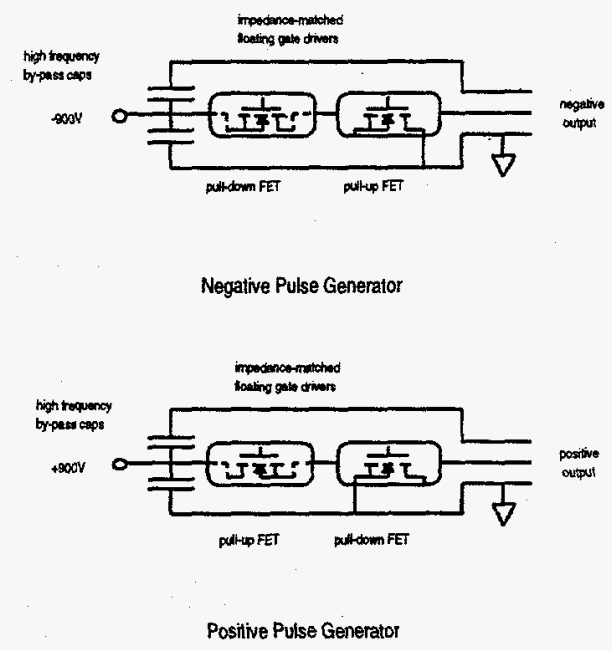

Figure 5: Simplified FET-based chopper-pulse generators.

\section{SUMMARY}

From considerations above we can conclude that at least two traveling-wave structures - the coax-plate design and the meander line, both with additional separators, can be used as the chopper current structure with the required rise and fall times in the 1-ns range. The measurement results for the prototype meander structure agree well with our computations, and further simulations will be performed to optimize a final design.

The existing FET technology for the pulse modulator is capable of providing a 4-ns rise time. Some development of fast power FET semiconductors should permit the desired 2.5-ns device.

The authors would like to gratefully acknowledge useful discussions with R.K. Cooper and G.R. Lambertson.

\section{REFERENCES}

[1] B.R. Appleton, et al., in Proceed. of EPAC (Barcelona, 1996), p.575 - IOP Publishing, Bristol, 1996.

[2] J.S. Lundsford and R.A. Hardekopf, IEEE Trans. NS30, 2830 (1983).

[3] M. Bartsch, et al., Computer Physics Comm. 72, 22 (1992)

[4] The notched-line approach was suggested to the authors by G.R. Lambertson, LBNL (March 1997). 